\title{
Moderate aortic stenosis: a new actor has come into stage
}

\author{
Adriana Postolache ${ }^{1}$, Julien Tridetti ${ }^{1}$, Mai-Linh Nguyen Trung ${ }^{1}$, Raluca Dulgheru ${ }^{1}$, Cécile Oury ${ }^{1}$, \\ Patrizio Lancellotti ${ }^{1,2}$
}

${ }^{1}$ University of Liège Hospital, GIGA Cardiovascular Sciences, Department of Cardiology and Cardiovascular Surgery, CHU SartTilman, Liège, Belgium; ${ }^{2}$ Gruppo Villa Maria Care and Research, Maria Cecilia Hospital, Cotignola, and Anthea Hospital, Bari, Italy

Correspondence to: Prof. Patrizio Lancellotti, MD, PhD. Department of Cardiology, University of Liège Hospital, Domaine Universitaire du Sart Tilman - B.35 - 4000 Liège, Belgium. Email: plancellotti@chuliege.be; plancellotti@chu.ulg.ac.be.

Comment on: Strange G, Stewart S, Celermajer D, et al. Poor Long-Term Survival in Patients With Moderate Aortic Stenosis. J Am Coll Cardiol 2019;74:1851-63.

Submitted Apr 16, 2020. Accepted for publication May 08, 2020.

doi: 10.21037/jtd-2020-48

View this article at: http://dx.doi.org/10.21037/jtd-2020-48

It is well established that patients with severe aortic stenosis (AS) have a poor prognosis once symptoms develop. European and American guidelines both recommend surgical (SAVR) or transcatheter (TAVR) aortic valve replacement in severe AS in the presence of symptoms or left ventricular systolic dysfunction, defined as a left ventricular ejection fraction (LVEF) $<50 \%(1,2)$. However, controversy still exists regarding the optimal timing of intervention in asymptomatic patients with severe AS, as several studies have shown that even truly asymptomatic patients based on an exercise test can have an increased risk of adverse events. Significant advances in SAVR and TAVR made in recent years have paved the way for large randomized trials that are currently evaluating the potential benefits of an early intervention in asymptomatic patients with severe AS (AVATAR, EvolVeD, ESTIMATE, EARLY-TAVR). Pending the results of these trials, staging the AS based on the evaluation of cardiac structural and hemodynamic changes as a whole, integrating information from rest and exercise echocardiography, cardiac magnetic resonance imaging (CMR) and biomarkers profile, can provide additional clues to the better understanding of the severity of the disease (3-5). In recent years, several small studies have shown that mild and moderate AS was not a benign disease, as initially envisioned, being associated with an increased incidence of cardiovascular events (6). As an example, Delesalle et al. reported an increased mortality rate $(47 \% \pm 3 \%)$ at 6 years of follow-up in patients with moderate AS and preserved LVEF (7). Patients with moderate AS and $\mathrm{LV}$ dysfunction appeared to have an even worse prognosis, with a $61 \%$ risk of adverse events (death, aortic valve replacement and heart failure hospitalization) at 6 years (8). In the HAVEC registry, concerning a larger cohort of patients with moderate AS, Lancellotti et al. reported a $2-, 4-$, and 8 -year survival rate of $94 \% \pm 1 \%, 89 \% \pm 2 \%$, and $78 \% \pm 4 \%$, respectively. Independent determinants of cardiovascular mortality were body surface area, dyslipidemia, peak aortic jet velocity, LVEF and aortic valve intervention. A peak aortic jet velocity $>3.5 \mathrm{~m} / \mathrm{s}$ and a LVEF $<60 \%$ were the predicting cut-off points associated with a worse outcome (9).

A recent study by Strange et al. appraised the shortand long-term outcome of more than 240,000 patients with mild, moderate and severe AS form the National Echocardiography Data of Australia (NEDA) registry (10). In this study, there was a significant and gradual increase in the risk of long-term mortality from mild to severe AS (adjusted hazard ratio from 1.44 to 2.09), supporting the consideration of AS as a disease continuum. But perhaps the most important observation from this trial was that, while the risk of long-term death in patients with mild AS was close to the risk observed in patients without AS, the risk of death in patients with moderate AS was close to that seen in patients with severe AS (5-year mortality of $56 \%$ and $67 \%$, respectively). In addition, the authors observed a marked increase in the risk of death from all causes and cardiovascular disease once the mean $\mathrm{AV}$ gradient was $>20 \mathrm{mmHg}$, even after adjustment for age, sex, LV systolic or diastolic dysfunction, and aortic regurgitation (10).

The question that comes to our mind is why these 
patients with moderate AS have a higher risk of cardiovascular complications? Discordant grading is a common problem in AS and it is conceivable that some of the patients included in the study of Strange et al. and classified as having a moderate AS group actually had a severe AS (11). However, inherent technical limitations or possible measurement errors alone cannot explain such a significant increase in the mortality risk. In their study, the authors mainly used the mean gradient and the maximum velocity for grading AS severity, which are known to be highly flow-dependent and may therefore underestimate AS severity in presence of low flow states. In such conditions, the aortic valve area may be superior to the peak velocity or mean gradient to assess AS severity and predict prognosis (9). To avoid this pitfall, the authors used the aortic valve area in patients with low-flow AS. Interestingly, they obtained the same AS classification when using the dimensionless index. Another possible reason is the lack of follow-up information, which is linked to the observational nature of this registry. Indeed, there were no data on disease progression or on any changes on symptomatic status between the index echocardiography until the time of death or census and it is possible that a significant proportion of patients who had moderate AS at baseline had progressed to severe AS and developed symptoms before death. We know that there is substantial variability in the rate of hemodynamic progression of AS and rapid progression to severe AS is not uncommon in patients with moderate AS (6). In addition, there were no data about the presence of coronary artery disease, atrial fibrillation or other comorbidities, which could have had an impact on the reported mortality. In previous, smaller studies, the incidence of death or cardiovascular events in patients with moderate AS was strongly influenced by the presence of comorbidities (6-8).

Beyond the few limitations mentioned above, which the authors have acknowledged, the study by Strange $e t$ al. confirms that moderate AS is not a mild intermediate condition. All this raises the question of the possible benefits of earlier intervention in these patients, with the aim of reducing cardiac damage and therefore improving the long-term prognosis after intervention. If the results of the studies on the impact of earlier AVR in asymptomatic patients with severe AS (AVATAR, EvolVeD, ESTIMATE, EARLY-TAVR) are positive, the conduct of a large randomized trial in this group of patients would allow us to better understand how to manage patients with moderate AS. Meanwhile, unlike current recommendations for watchful waiting and echocardiographic monitoring at relatively long intervals $(1,2)$, a better approach would be to assess the stage of the disease as in severe AS by integrating a set of clinical, biological and imaging data (3-5). Although the risk stratification of patients with moderate AS is still in its infancy, several parameters could help identify patients requiring more frequent follow-up or earlier intervention. A proposed follow-up plan for patients with moderate AS is shown in Figure 1.

Due to its wide availability, echocardiography should remain the main investigation used in the follow-up of these patients, but we must consider broadening our vision and take into consideration not only the changes in the valve disease (severity of AS, degree and extent of valve calcification) but also of the heart as a whole. The assessment of LV function plays an important role in the evaluation of these patients. It appears that the drop in LVEF occurs before AS becomes severe (12). In patients with moderate or severe AS, a LVEF $<60 \%$ and a global longitudinal strain (GLS) $<14.7 \%$ are predictors of a worse outcome, even after SAVR $(9,12,13)$. These patients could therefore benefit from more frequent follow-up or an earlier intervention. Noteworthy, patients with moderate AS and a $\mathrm{LVEF}<50 \%$ due to myocardial damage related to ischemic or non-ischemic cardiomyopathy have an increased risk of adverse events (8). In this context, even the presence of moderate AS can significantly contribute to the symptomatic status, and the overall LV afterload and LV dysfunction. In these patients considered to be at higher risk, SAVR is associated with increased operative mortality (14). The ongoing TAVR-UNLOAD trial is currently comparing the efficacy and safety of TAVR in addition to optimal heart failure therapy (OHFT) to OHFT alone in patients with moderate AS and reduced LVEF.

Exercise echocardiography can also help guide the appropriate clinical follow-up in patients with moderate AS and preserved LVEF. An exercise-increase in mean aortic pressure gradient $>20 \mathrm{mmHg}$ identifies patients with a faster progression of the stenosis severity (15), and, in those with a mean rest gradient $>35 \mathrm{mmHg}$, an increased risk of death or need for AVR (16). Patients with these characteristics should also benefit from a closer follow-up (every 6 months).

The evaluation of myocardial fibrosis with CMR can also complement the initial echocardiographic evaluation of patients with moderate AS. The degree of myocardial fibrosis correlates with the disease progression and the risk of adverse events in AS. Mid-wall fibrosis detected by late gadolinium enhancement (LGE) is an independent 


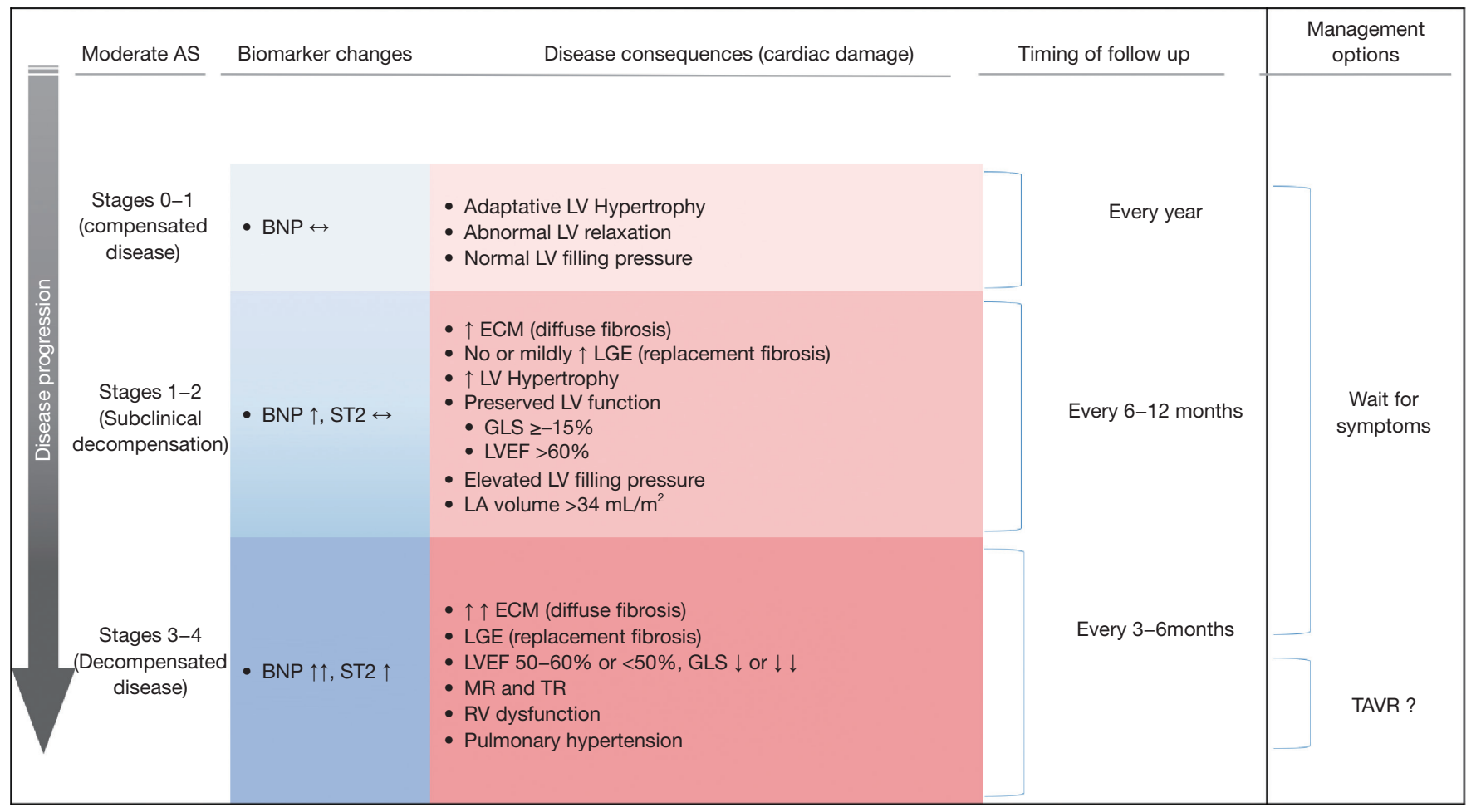

Figure 1 Staging and proposed follow-up plan in moderate aortic stenosis. The staging approach shown based on the presence of cardiac damage allows for the risk assessment of the individual patient with moderate AS and determines the timing of follow-up. BNP, brain natriuretic peptide; ECM, extracellular matrix assessed with CMR imaging T1 mapping; GLS, global longitudinal strain assessed by echocardiography using speckle-tracking; LA, left atrium; LGE, late gadolinium enhancement assessed with CMR; LV, left ventricular; LVEF, left ventricular ejection fraction; MR, mitral regurgitation; RV, right ventricle; ST2, suppression of tumorigenicity 2 (marker of cardiac remodeling and myocardial fibrosis); TR, tricuspid regurgitation.

predictor of mortality in patients with moderate or severe AS, having incremental prognostic value compared to LVEF (17). Moreover, diffuse myocardial fibrosis (DMF) by native T1 mapping is an early phenomenon, preceding focal scarring and a high native $\mathrm{T} 1$ value identifies patients at increased risk of clinical events, both pre- and postoperatively, and is an independent predictor of outcome in addition to LGE (18).

B-type natriuretic peptide (BNP) is probably the biomarker most frequently measured in AS; it is predominantly secreted by ventricular cardiac myocytes in response to increased wall stress. BNP levels correlate with the symptomatic status and the severity of AS and predict symptomatic deterioration and adverse clinical outcomes during follow-up (19). In a recent study in patients with moderate AS, a higher NT-pro BNP value $(>888 \mathrm{pg} / \mathrm{dL})$ was significantly associated with an increase in all-cause mortality and cardiovascular death, even among those undergoing AVR during follow-up and after adjustment for comorbidity or echocardiographic parameter such as LVEF and E/e' (20). Patients with moderate AS and increased BNP levels might potentially benefit from closer followedup or earlier intervention, and changes in serial BNP values might provide additional information on individual clinical outcome (21).

In conclusion, patients with moderate AS constitute a highly heterogeneous group with regard to the progression rate of the disease and risk of adverse events. Hence, optimizing risk stratification process using a multiparametric approach and criteria identified to be of prognostic value may be useful to adjust the frequency of follow-up visits of these patients. Those being at higher risk on the basis of these criteria should receive a closer and more frequent follow-up than that (every 2 years) recommended in societal guidelines and might benefit from an early AVR. 


\section{Acknowledgments}

Funding: None.

\section{Footnote}

Provenance and Peer Review: This article was commissioned and reviewed by the Section Editor Hui-Ping Zhang, MD (Department of Cardiology, Beijing Hospital, the Fifth Affiliated Hospital of Peking University, Beijing, China).

Conflicts of Interest: All authors have completed the ICMJE uniform disclosure form (available at http://dx.doi. org/10.21037/jtd-2020-48). The authors have no conflicts of interest to declare.

Ethical Statement: The authors are accountable for all aspects of the work in ensuring that questions related to the accuracy or integrity of any part of the work are appropriately investigated and resolved.

Open Access Statement: This is an Open Access article distributed in accordance with the Creative Commons Attribution-NonCommercial-NoDerivs 4.0 International License (CC BY-NC-ND 4.0), which permits the noncommercial replication and distribution of the article with the strict proviso that no changes or edits are made and the original work is properly cited (including links to both the formal publication through the relevant DOI and the license). See: https://creativecommons.org/licenses/by-nc-nd/4.0/.

\section{References}

1. Baumgartner H, Falk V, Bax JJ, et al. 2017 ESC/EACTS Guidelines for the management of valvular heart disease. Eur Heart J 2017;38:2739-91.

2. Nishimura RA, Otto CM, Bonow RO, et al. 2017 AHA/ ACC Focused Update of the 2014 AHA/ACC Guideline for the Management of Patients With Valvular Heart Disease: A Report of the American College of Cardiology/ American Heart Association Task Force on Clinical Practice Guidelines. Circulation 2017;135:e1159-95.

3. Tastet L, Tribouilloy C, Maréchaux S, et al. Staging Cardiac Damage in Patients With Asymptomatic Aortic Valve Stenosis. J Am Coll Cardiol 2019;74:550-63.

4. Vannan MA, Pibarot P, Lancellotti P. Aortic Stenosis: The Emperor's New Clothes. J Am Coll Cardiol 2019;74:1864-7.
5. Lancellotti P, Vannan MA. Timing of intervention in aortic stenosis. N Engl J Med 2020;382:191-3.

6. Rosenhek R, Klaar U, Schemper M, et al. Mild and moderate aortic stenosis. Natural history and risk stratification by echocardiography. Eur Heart J 2004;25:199-205.

7. Delesalle G, Bohbot Y, Rusinaru D, et al. Characteristics and Prognosis of Patients With Moderate Aortic Stenosis and Preserved Left Ventricular Ejection Fraction. J Am Heart Assoc 2019;8:e11036.

8. van Gils L, Clavel MA, Vollema EM, et al. Prognostic Implications of Moderate Aortic Stenosis in Patients With Left Ventricular Systolic Dysfunction. J Am Coll Cardiol 2017;69:2383-92.

9. Lancellotti P, Magne J, Dulgheru R, et al. Outcomes of Patients With Asymptomatic Aortic Stenosis Followed Up in Heart Valve Clinics. JAMA Cardiol 2018;3:1060-8.

10. Strange G, Stewart S, Celermajer D, et al. Poor LongTerm Survival in Patients With Moderate Aortic Stenosis. J Am Coll Cardiol 2019;74:1851-63.

11. Berthelot-Richer M, Pibarot P, Capoulade R, et al. Discordant Grading of Aortic Stenosis Severity: Echocardiographic Predictors of Survival Benefit Associated With Aortic Valve Replacement. JACC Cardiovasc Imaging 2016;9:797-805.

12. Ito S, Miranda WR, Nkomo VT, et al. Reduced Left Ventricular Ejection Fraction in Patients With Aortic Stenosis. J Am Coll Cardiol 2018;71:1313-21.

13. Magne J, Cosyns B, Popescu BA, et al. Distribution and Prognostic Significance of Left Ventricular Global Longitudinal Strain in Asymptomatic Significant Aortic Stenosis: An Individual Participant Data Meta-Analysis. JACC Cardiovasc Imaging 2019;12:84-92.

14. Clavel MA, Fuchs C, Burwash IG, et al. Predictors of outcomes in low-flow, low-gradient aortic stenosis: results of the multicenter TOPAS Study. Circulation 2008;118:S234-42.

15. Ringle A, Levy F, Ennezat PV, et al. Relationship between exercise pressure gradient and haemodynamic progression of aortic stenosis. Arch Cardiovasc Dis 2017;110:466-74.

16. Maréchaux S, Hachicha Z, Bellouin A, et al. Usefulness of exercise-stress echocardiography for risk stratification of true asymptomatic patients with aortic valve stenosis. Eur Heart J 2010;31:1390-7.

17. Dweck MR, Joshi S, Murigu T, et al. Midwall fibrosis is an independent predictor of mortality in patients with aortic stenosis. J Am Coll Cardiol 2011;58:1271-9.

18. Lee H, Park JB, Yoon YE, et al. Noncontrast Myocardial 
T1 Mapping by Cardiac Magnetic Resonance Predicts Outcome in Patients With Aortic Stenosis. JACC

Cardiovasc Imaging 2018;11:974-83.

19. Lancellotti P, Moonen M, Magne J, et al. Prognostic effect of long-axis left ventricular dysfunction and B-type natriuretic peptide levels in asymptomatic aortic stenosis. Am J Cardiol 2010;105:383-8.

Cite this article as: Postolache A, Tridetti J, Nguyen Trung ML, Dulgheru R, Oury C, Lancellotti P. Moderate aortic stenosis: a new actor has come into stage. J Thorac Dis 2020;12(11):7064-7068. doi: 10.21037/jtd-2020-48
20. Ito S, Miranda WR, Jaffe AS, et al. Prognostic Value of N-Terminal Pro-form B-Type Natriuretic Peptide in Patients With Moderate Aortic Stenosis. Am J Cardiol 2020;125:1566-70.

21. Henri C, Magne J, Dulgheru R, et al. Usefulness of serial B-type natriuretic peptide assessment in asymptomatic aortic stenosis. Am J Cardiol 2014;114:441-8. 